\section{(6) OPEN ACCESS}

\title{
Estimates of peripartum and postnatal mother-to-child transmission probabilities of HIV for use in Spectrum and other population-based models
}

\author{
Nigel Rollins, ${ }^{1,2}$ Mary Mahy, ${ }^{3}$ Renaud Becquet, ${ }^{4,5}$ Louise Kuhn, ${ }^{6}$ Tracy Creek, ${ }^{7}$ \\ Lynne Mofenson ${ }^{8}$
}

- Additional data are published online only. To view these files please visit the journal online (http://dx.doi.org/10.1136/ sextrans-2012-050709).

1 Department of Maternal, Newborn, Child and Adolescent Health, World Health Organization, Geneva, Switzerland

${ }^{2}$ Department of Paediatrics, University of KwaZulu-Natal, Durban, South Africa

${ }^{3}$ Department of Evidence, Innovation and Policy, Joint United Nations Programme on HIV/AIDS (UNAIDS), Geneva, Switzerland

${ }^{4}$ Department of INSERM, unité 897, Centre de recherche 'Epidémiologie et Biostatistique', Bordeaux, France

${ }^{5}$ Institut de Santé Publique Epidémiologie Développement (ISPED), Université Victor Segalen Bordeaux 2, Bordeaux, France

${ }^{6}$ Gertrude H. Sergievsky Center, College of Physicians and Surgeons, Columbia University, New York, New York, USA ${ }^{7}$ Children's Healthcare of Atlanta, Atlanta, Georgia ${ }^{8}$ Center for Research for Mothers and Children, National Institute of Child Health and Human Development, National Institutes of Health, Rockville, Maryland, USA

\section{Correspondence to}

Dr Nigel Rollins, Department of Maternal, Newborn, Child and

Adolescent Health, World Health Organization, Avenue Appia 20, Geneva 1211

Switzerland;

rollinsn@who.int

\section{UNAIDS Report 2012 \\ Guest Editors \\ Karen Stanecki \\ Peter D Ghys \\ Geoff P Garnett \\ Catherine Mercer}

Accepted 24 September 2012

\section{ABSTRACT}

Background The Global Plan Towards the Elimination of New HIV Infections among Children and Keeping Their Mothers Alive aims to reduce by 2015 the number of new infections in children, in 22 priority countries, by at least 90\% from 2009 levels. Mathematical models, such as Spectrum, are used to estimate national and global trends of the number of infants infected through motherto-child transmission (MTCT). However, other modelling exercises have also examined MTCT under different settings. MTCT probabilities applied in models to populations that are assumed to receive antiretroviral interventions need to reflect the most current risk estimates.

Methods The UNAIDS Reference Group on Estimates, Modelling and Projections held a consultation to review data on MTCT probabilities. Published literature, recent conferences and data from personal communications with principle investigators were reviewed. Based on available data, peripartum and postnatal transmission probabilities were estimated for different antiretroviral drug regimens and maternal CD4 levels including for women with incident infection.

Results Incident infections occurring during pregnancy are estimated to be associated with a $30 \%$ probability of MTCT; incident infections during breast feeding lead to a $28 \%$ probability of postnatal MTCT. The 2010 WHO recommended regimens (Options A or B) are estimated to be associated with a $2 \%$ peripartum transmission probability and $0.2 \%$ transmission probability per month of breast feeding. Peripartum and postnatal transmission probabilities were lowest for women who were taking antiretroviral therapy before the pregnancy namely $0.5 \%$ peripartum and $0.16 \%$ per month of breast feeding, respectively.

Discussion These updated probabilities of HIV transmission (applied to Spectrum in April 2011) will be used to estimate new child HIV infections and track progress towards the 2015 targets of the Global Plan.

\section{INTRODUCTION}

Modelling approaches are routinely used to estimate national and global trends of the number of infants infected through mother-to-child transmission of HIV (MTCT). ${ }^{12}$ Such models depend on input data from individual countries such as the number of HIV-positive women receiving antiretroviral drug (ARV) interventions. ${ }^{3} 4$ Models apply HIV transmission probabilities (both peripartum and postnatal) by ARV intervention and by timing of maternal infection (either incident or prevalent) to derive population-based HIV infection rates.

WHO guidelines for preventing MTCT are updated to reflect the most current research evidence and recommend the most effective and safe interventions. The $2010 \mathrm{WHO}$ guidelines for ARVs for treating pregnant women and preventing HIV infection in infants introduced several important revisions and, for the first time, recommended ARVs to prevent postnatal transmission of HIV through breast feeding. ${ }^{5}$ These guidelines presented two main ARV regimens, namely Options $\mathrm{A}$ and $\mathrm{B}$, which both include starting HIV-infected women with CD4 counts less than 350 cells $/ \mathrm{ml}$ on lifelong antiretroviral therapy (ART). In Option A, women not eligible for ART should receive daily zidovudine (AZT) from the first trimester until delivery and, in breastfeeding populations, HIV-exposed infants should receive daily nevirapine until 1 week after all breast feeding. In Option B, women not eligible for ART should receive daily triple ARVs from the first trimester until delivery and, in breastfeeding populations, continue with these ARVs until 1 week after all breast feeding. In non-breastfeeding populations, both options recommend that HIV-exposed infants receive ARV prophylaxis for the first 6 weeks post partum.

The Global Plan Towards the Elimination of New HIV Infections among Children and Keeping Their Mothers Alive has set a target that by 2015 the number of new HIV infections in children will be reduced, in each of 22 priority countries, ${ }^{\mathrm{i}}$ by at least 90\% from 2009 levels. ${ }^{6}$ The Plan has also set a target of reducing HIV-associated pregnancy-related deaths by $50 \%$ in the same countries. Progress towards these targets is modelled using the AIDS Impact Module (AIM) within the Spectrum demographic modelling package.

In order to track progress towards the targets of the Global Plan, MTCT probabilities applied to populations in models need to reflect the most current transmission risk estimates. ${ }^{4}$ In particular, models need to include the probability of transmission in children due to new incident infection among pregnant and lactating women, and reflect the potential impact of ARV interventions that reduce transmission through breast feeding. Here we

Angola, Botswana, Burundi, Cameroon, Chad, Côte d'Ivoire, Democratic Republic of the Congo, Ethiopia, Ghana, India, Kenya, Lesotho, Malawi, Mozambique, Namibia, Nigeria, South Africa, Swaziland, Uganda, United Republic of Tanzania, Zambia and Zimbabwe. 
present the evidence for the revised transmission probabilities applied in Spectrum since April 2011.

\section{How Spectrum uses the transmission probabilities}

The transmission probabilities described in this paper were specifically intended for use in the Spectrum computer package or other similar models that estimate the impact of prevention of mother-to-child transmission of HIV (PMTCT) programmes. A detailed description of Spectrum is available elsewhere. ${ }^{4}$ The peripartum transmission probabilities are applied to all deliveries among HIV-positive women to estimate the number of peripartum infections. The postnatal transmission probabilities are applied to the estimated proportion of HIV-negative children who are breast feeding among HIV-positive mothers. Postnatal transmission is calculated based on the per cent of women still breast feeding by months since birth. A monthly transmission probability is applied for each month that the child is breast feeding (see figure 1).

\section{METHODS}

The probabilities of peripartum and postnatal MTCT of HIV according to ARV regimen and maternal CD4 count among women with prevalent infection and among women with incident infection were reviewed for a meeting that aimed to update modelling approaches and assumptions used in Spectrum that was held in Washington, DC, September 2010. A process to agree on estimates of transmission probability was agreed by the authors following a critical review of available evidence. While the initial meeting was held in September 2010, data from studies reported in peer-reviewed journals from 1990 until the beginning of 2012 were considered for inclusion.

\section{Studies included in the analysis}

Data used for this analysis included published literature, conference presentations and data from personal communication with researchers when data were not otherwise available. The authors reviewed these data for quality, internal consistency and relevance. Data from randomised studies were prioritised but cohort and observational data were also included providing the reports indicated rigorous study methods, appropriate sample sizes and provided direct transmission estimates from relevant study populations. Details of these reports are summarised in an annotated bibliography (see working paper in online supplementary appendix).

\section{Definitions and assumptions underlying estimates of HIV transmission probabilities}

Peripartum HIV transmission reflects combined in utero and intrapartum transmission and is measured by HIV status of infants at 4-6weeks of age. It assumes no additional early transmission due to breast feeding. In non-breastfed infants, HIV status of infants at 4-6 weeks or any time thereafter would represent peripartum transmission. In breastfed infants, any additional transmission that occurs after 6 weeks of age would be regarded as postnatal transmission and attributable to breast feeding.

Postnatal HIV transmission reflects infections in infants or children who were HIV uninfected through pregnancy and delivery (identified through a negative HIV test at 4-6 weeks) and who subsequently become HIV-infected while breast feeding.

Postnatal transmission rates captured for each study were either those directly reported by investigators, even if the age at which peripartum transmission was measured differed between studies namely 2, 4, 6 or 8 weeks post partum, or were calculated from data included in the reports. Some investigators reported point or cumulative transmission at birth, 6 weeks and other postnatal time points such as 3, 6, 12 and 18 months. In these situations, HIV transmission due to breast feeding was estimated by subtracting HIV infections (or transmission rates) among 6-week-old HIV-exposed infants from HIV infections (or transmission rates) among HIV-exposed breastfeeding infants identified any time thereafter.

Depending on data provided, monthly postnatal transmission probabilities were calculated by dividing the cumulative transmission per cent measured over a particular time interval by the number of months in that time period minus 4 weeks. Four weeks was deducted from the breastfeeding exposure period as HIV DNA laboratory assays only reliably detect infections that occur up to about 4 weeks prior to measurement. For example, if transmission was measured at 6 months, then the breastfeeding exposure period was deemed to be 1 month less, or 5 months. If the transmission rates at 6 weeks and 6 months were $5 \%$ and $8.5 \%$, respectively, then $3.5 \%$ HIV transmission would be attributed to 5 months of breast feeding, or $0.7 \%$ per month of breast feeding.

Alternatively, if studies reported the median breastfeeding period as less than the time at which HIV status was determined, then the cumulative transmission per cent by the time of measurement was divided by the median duration of breast feeding. For example, if postnatal transmission was estimated to be $9 \%$ at 12 months with a median breastfeeding period of 7 months, then the monthly transmission probability

Figure 1 Calculating new HIV infections among children in Spectrum. 
associated with breast feeding would be $9 / 7=1.29 \%$ per month of breast feeding.

This simple, and easily applied, calculation does not account for the conditional probability of postnatal infection, for example, the conditional probability that the child is not infected in the previous month or at birth, which will have a significant impact when multiplied over several months of exposure or to large probabilities. Spectrum currently only includes two decimal spaces of accuracy for the monthly transmission probabilities. Thus, a more accurate calculation (see example in footnote ${ }^{\mathrm{ii}}$ ) would have no impact on the overall rates in the current model.

Incident infection refers to newly acquired infections in pregnant or lactating women. Methods used for identifying these women were different between studies and the investigators' approach for inclusion of this population was accepted. Incident infection was usually identified through seroconversion of women who previously tested negative for HIV. Transmission probabilities were not disaggregated by maternal CD4 count as, although women with incident infection have high viral load similar to women with longstanding HIV infection, their CD4 counts are not depleted in the early stages of infection.

We did not estimate a monthly transmission probability for lactating women with incident HIV infection as the reported high risks of transmission, associated with high viral load in mothers during primary viraemia, may only be present for 1-2 months. It was considered inappropriate to apply an average monthly probability over a duration of breast feeding, whether long or short, within a model.

$A R V$ regimens were defined according to $\mathrm{WHO}$ recommendations that were valid at the time of respective studies. The term WHO 2006 dual prophylaxis is applied to a range of ARV interventions that were included in the recommendations at that time. This included settings in which breast feeding was the dominant infant feeding practice and also settings where replacement feeding (formula feeds) were the default recommendation. It also includes settings where single dose nevirapine may or may not have been given to infants in the immediate postnatal period (within $72 \mathrm{~h}$ ). While not included in WHO recommendations at that time, ${ }^{7}$ this practice was reported in some studies.

Any breast feeding includes exclusive, predominant and partial breast feeding (mixed breast feeding=predominant and partial breast feeding). No attempt to further disaggregate was made given limitations in the data.

\section{Estimating HIV transmission probabilities}

The median peripartum and postnatal transmission probability and ranges for each ARV regimen and CD4 count were determined from reported data. Exceptions to this approach are separately presented and justified. In some instances, the median value was overruled based on other considerations and in these situations the rationale is outlined. The transmission probabilities were not weighted by the size of the study as each study had its own limitations.

\footnotetext{
ii An example of calculating the conditional probability: if the 4-6 week risk is $5 \%$ and the 6 -month total risk is $10 \%$ then the risk of postnatal infection is $10 \%$ minus $5 \%$ divided by those infants who were negative at the start of the time period so $(10-5) /(100-5)$. Convert the cumulative risk into a monthly rate $[-\ln (1-P))] / t$ where $\mathrm{P}$ is the conditional probability and $t$ is the number of months of exposure. Calculate back to a monthly probability $1-\mathrm{e}^{\wedge}(-\mathrm{r})$ where $\mathrm{r}$ is the monthly rate calculated in the first step.
}

When reports presented transmission probabilities separately for mothers with CD4 counts $350-500$ cells $/ \mathrm{ml}$ and $>500$ cells $/ \mathrm{ml}$, these probabilities were averaged and included as the transmission probability for women with CD4 counts $>350$ cells $/ \mathrm{ml}$. This concept was applied similarly in some instances for other grouped ranges of CD4 counts (see online supplementary table 1).

For the purposes of Spectrum it was not necessary to calculate the transmission probabilities for different $\mathrm{CD} 4$ distributions for single dose nevirapine (sdNVP) and WHO 2006 dual ARV regimens as these interventions will only be applied to historical populations without reference to CD4 counts when disaggregated data by CD4 distributions were not generally available. Options A and B were only recommended for women with CD4 counts of 350 or higher and thus probabilities for those scenarios were estimated. Although not recommended by WHO for women with CD4 counts below 350 cells $/ \mathrm{ml}$, a transmission probability is included for Option A in this population because (i) those data were available from some reports and (ii) some countries were providing Option A to this population before revisions of national guidelines (ie, criteria for ART from a CD4 count of $<200$ to $<350$ ) and (iii) in settings where CD4 testing is not routinely available, some women with lower CD4 counts may only receive this intervention.

Monthly postnatal transmission probabilities were not estimated for a population where CD4 count is 'not specified' (as presented in the first column of table 1 for Peripartum transmission). For modelling postnatal transmission in populations where data on maternal CD4 counts are not known, such as historical cohorts, then assumptions can be made on the distribution of CD4 counts in these populations and an average transmission probability is secondarily estimated and attributed. Since 2011 Spectrum assumes a CD4 distribution among pregnant women to determine which transmission probability will be applied during breast feeding.

Separate monthly postnatal transmission probabilities were not estimated for populations receiving sdNVP or WHO 2006 dual prophylaxis as it was assumed that, although both regimens provide an ARV to infants postnatally, neither is considered to have substantial impact on postnatal transmission through breast feeding.

\section{RESULTS}

Table 1 presents the estimated transmission probabilities by ARV regimen and CD4 level. Details of transmission probabilities reported in each study are presented in the online supplementary table 1. An annotated bibliography of studies is included in the working paper (online supplementary appendix).

\section{Transmission probabilities due to incident HIV infection}

Four reports provided data on peripartum transmission ${ }^{8-11}$ and six reports on postnatal transmission ${ }^{12-17}$ among women who had become infected during pregnancy or lactation. The reported transmission probability for peripartum transmission ranged from $13 \%$ to $30 \%$. The high value of the peripartum range, that is, $30 \%$ was selected in light of other reports that documented high odds ratios for infant transmission associated with maternal incident infection in pregnancy but did not provide transmission probabilities. For postnatal transmission, the range was $14.3 \%-56 \%$ with a median of $28 \%$. These are comparable with transmission probabilities to infants born to mothers with $\mathrm{CD} 4<200 \mathrm{cell} / \mathrm{s} / \mathrm{ml}$, not receiving any treatment, and who have high viral load. 
Table 1 Summary of transmission probabilities by antiretroviral regimen and maternal CD4 count

\begin{tabular}{|c|c|c|c|c|c|c|}
\hline \multirow[b]{2}{*}{ Regimen } & \multicolumn{4}{|l|}{ Peripartum transmission } & \multicolumn{2}{|c|}{$\begin{array}{l}\text { Postnatal transmission per month of any } \\
\text { BF* (except incident infection) }\end{array}$} \\
\hline & CD4 count not specified & CD4 $<200$ & CD4 200-350 & CD4 $350+$ & CD4 $<350$ & CD4 $>350$ \\
\hline $\begin{array}{l}\text { Incident infections (range of } \\
\text { reported transmission probabilities) }\end{array}$ & $30 \%(13 \%-30 \%)^{6-9}$ & & & & $28 \%(14.3 \%-56 \%)^{10-15}$ & \\
\hline $\begin{array}{l}\text { No prophylaxis (range of reported } \\
\text { transmission probabilities) }\end{array}$ & $22 \%(15 \%-25 \%)^{16-20}$ & $\begin{array}{l}37 \% \\
(22 \%-54 \%)^{182122}\end{array}$ & $\begin{array}{l}27 \% \\
(13.1 \%-32.6 \%)^{18} 21\end{array}$ & $\begin{array}{l}15 \% \\
(9.7 \%-20.2 \%)^{182122}\end{array}$ & $1.57 \% / \mathrm{mBF}^{23-25}$ & $0.51 \% / \mathrm{m} \mathrm{BF}^{23-252930}$ \\
\hline $\begin{array}{l}\text { sdNVP (range of reported } \\
\text { transmission probabilities) }\end{array}$ & $12 \%(9.4 \%-12.1 \%)^{31-34}$ & & & & $1.57 \% / \mathrm{mBF}^{23-25}$ & $0.51 \% / \mathrm{m} \mathrm{BF}^{23-252930}$ \\
\hline $\begin{array}{l}\text { WHO } 2006 \text { dual prophylaxis (range } \\
\text { of reported transmission } \\
\text { probabilities) }\end{array}$ & $\begin{array}{l}4 \% \\
(2.3 \%-5.3 \%)^{29} 3035-37\end{array}$ & & & & $1.57 \% / \mathrm{m} \mathrm{BF}^{23-25}$ & $0.51 \% / \mathrm{m} \mathrm{BF}^{23-252930}$ \\
\hline Option Ał & & & $4 \% \dagger$ As WHO 2006 & $2 \%^{24} 2936$ & & $0.2 \% / \mathrm{m} \mathrm{BF}^{39} 40$ \\
\hline Option B§ & & & & $\begin{array}{l}2 \% \\
(0.9 \%-2.9 \%)^{24} 41-44\end{array}$ & & $0.2 \% / \mathrm{m} \mathrm{BF}^{24} 3943-45$ \\
\hline $\begin{array}{l}\text { ART (range of reported } \\
\text { transmission probabilities) }\end{array}$ & & $2 \% 2429$ & $3041-43$ & & $0.2 \% / \mathrm{m} \mathrm{BF}^{29} 3043-45$ & \\
\hline ART (before pregnancy) & & $0.5 \%{ }^{24} 4$ & 12464748 & & $0.16 \% / \mathrm{m} \mathrm{BF}^{24} 394344$ & \\
\hline
\end{tabular}

Shading indicates transmission probabilities that are not estimated for a particular regimen either because the regimen is not recommended for women with a particular CD4 count, for example, Option A or B for women with CD4 counts less than 350 cells/ml, or because transmission data were not available for a regimen by CD4 count, for example, sdNVP in women with CD4 350-500 cells/ml.

${ }^{*}$ For the transmission probabilities associated with breast feeding the values are given to two decimal places since rounding these values up or down would result in significantly greater or lesser transmission rates when multiplied according to the duration of breast feeding.

tProviding Option A to breastfeeding mothers with CD4 counts 200-350 is not recommended. However, it is noted that this situation may arise in settings where systems to perform CD4 counts are not in place and women needing to be on lifelong ART are not being readily identified.

fIn Option A, HIV-positive pregnant women who are eligible for lifelong ART should be started on treatment in the first trimester of pregnancy. HIV-positive pregnant women who are not eligible for ART should receive daily AZT from 14 weeks gestation until delivery, single dose nevirapine during labour and AZT +3TC during labour and for 7 days post partum. HIV-exposed infants would receive AZT or NVP until 6 weeks of age and if breast feeding then NVP would continue until 1 week after all breast feeding has stopped.

$\S / n$ Option B, HIV-positive pregnant women who are eligible for lifelong ART should be started on treatment in the first trimester of pregnancy. HIV-positive pregnant women who are not eligible for lifelong ART should receive one of four combinations of ARVs during pregnancy throughout the breastfeeding period and 1 week after. Exposed infants would receive either AZT or NVP for 1 week.

3TC, lamivudine; ART, antiretroviral therapy; ARV, antiretroviral drug; AZT, zidovudine; BF, breastfeeding; sdNVP, single dose nevirapine.

\section{Transmission probabilities in the context of no ARV prophylaxis}

Five studies reported peripartum transmission probabilities without disaggregating by CD4 count. ${ }^{18-22}$ While there were significant geographical variations, there was no clear relationship between transmission rates and either breastfeeding or non-breastfeeding populations. The range of transmission probabilities was $15.3 \%-25.5 \%$ with a median of $22 \%$. Four reports provided data on peripartum transmission among mothers with CD4 counts $<200$ cells/ml in east, west and southern Africa and also Thailand and France 2024 (Humphrey J, personal communication, 2010). In these populations, the range of transmission probability was $22.4 \%-54.2 \%$ with a median of $37 \%$. Among women with CD4 counts $200-350$ cells/ml, the range of transmission probabilities was $13.1-32.6 \%$ with a median of $27 \%^{20}{ }^{23}$ (Humphrey J, personal communication, 2010). Limited data were available from four reports to inform transmission probabilities in women with CD4 counts $>350$ cells/ml $\mathrm{ml}^{20} 2324$ (Humphrey J, personal communication, 2010). In one study, an average transmission probability was calculated for women with CD4 counts $>350$ cells $/ \mathrm{ml}$ at each site as described in the Methods section. ${ }^{23}$ The range of transmission probabilities was $9.7 \%-20.2 \%$ with a median value of $15 \%$.

With respect to postnatal transmission through breast feeding among mothers with CD4 count $<350^{25-27}$ (Humphrey J, personal communication, 2010). (Becquet $\mathrm{R}$, personal communication, 2010), when maternal CD4 count is not considered, the monthly postnatal transmission probability is about $0.8 \%$ per month of breast feeding ${ }^{28}$ or 9.2 infections per 100 child years. $^{29}$ Some studies only reported a three to ninefold increased risk as an OR or HR of transmission among mothers with lower CD4 count compared with those with higher CD4 counts. ${ }^{30}$
The range of monthly transmission probabilities in mothers with CD4 counts $<350$ cells/ml was $0.84 \%-2.5 \%$ per month of breast feeding and the median value was $1.57 \%$ per month of breast feeding. Seven reports provided postnatal transmission data among mothers with CD4 counts equal to or more than 350 cells $/ \mathrm{ml}^{25-27} 31 \quad 32$ ( $\mathrm{R}$ Becquet, Personal communication, 2010) (Humphrey J, personal communication, 2010). Transmission probabilities from the observational cohorts and the randomised control trial groups in the Kesho Bora study were not averaged as they were derived from distinct populations. The range of monthly transmission probabilities was $0.1 \%-0.96 \%$ per month of breast feeding with a median value of $0.51 \%$ per month of breast feeding.

\section{Transmission probabilities in the context of single dose nevirapine}

Peripartum transmission probabilities were noted in four reports with a range of $9.4 \%-12.1 \% .^{33-36}$ The median value was $12 \%$.

\section{Transmission probabilities in the context of WHO 2006 dual prophylaxis}

Peripartum transmission probabilities were noted in five reports with a range of $2.3 \%-5.3 \%$ and a median value of $4 \% .{ }^{31} 32$ 37-39

\section{Transmission probabilities in the context of Option A regimen}

No studies reported transmission probabilities when all components of the ARV interventions recommended in Option A were provided to the population for whom Option A is specifically recommended. Table 2 summarises the elements of Option A that are reported in each study. 
Table 2 Elements of Option A regimen reported in each study

\begin{tabular}{|c|c|c|}
\hline & $\begin{array}{l}\text { Interventions offered in } \\
\text { SWEN, }{ }^{36} \text { PEPI, }^{40} \text { BANS }^{41} \\
\text { and HPTN } 046^{42}\end{array}$ & $\begin{array}{l}2010 \text { WHO Option A for } \\
\text { breastfeeding communities }\end{array}$ \\
\hline $\begin{array}{l}\text { Antenatal ARVs to } \\
\text { pregnant HIV-infected } \\
\text { women }\end{array}$ & $\begin{array}{l}\text { Generally single dose NVP } \\
\text { to mother } \\
\text { Some mothers also started } \\
\text { on lifelong treatment }\end{array}$ & $\begin{array}{l}\text { AZT during pregnancy }+ \\
\text { sdNVP during labour }+ \\
\text { AZT/3TC from start of labour } \\
\text { until } 7 \text { days after }\end{array}$ \\
\hline $\begin{array}{l}\text { Time when ARVs started } \\
\text { antenatally }\end{array}$ & Onset of labour & AZT from 14 weeks \\
\hline Maternal CD4 count & $\begin{array}{l}\text { Included women with } \\
\text { CD4 counts as low as } \\
200 \text { cells } / \mathrm{ml}\end{array}$ & $\begin{array}{l}\text { Only for women with } \\
\text { CD4 <350 cells/ml }\end{array}$ \\
\hline $\begin{array}{l}\text { ARV intervention to } \\
\text { infants to prevent } \\
\text { peripartum transmission }\end{array}$ & sdNVP after delivery & NVP daily for 6 weeks \\
\hline $\begin{array}{l}\text { ARV intervention to } \\
\text { infants to prevent } \\
\text { postnatal transmission }\end{array}$ & $\begin{array}{l}\text { Nevirapine to infants while } \\
\text { breast feeding ( } 6 \text { weeks, } \\
14 \text { weeks or } 6 \text { months) }\end{array}$ & $\begin{array}{l}\text { Nevirapine to infants until } \\
1 \text { month after end of all } \\
\text { breast feeding }\end{array}$ \\
\hline
\end{tabular}

3TC, lamivudine; ARV, antiretroviral drug.

However, studies did provide data on individual components of Option A. In West Africa, among infants who were primarily given formula feeds and whose mothers had CD4 counts $>350$ and received AZT from either 28 or 32 weeks + lamivudine (3TC) and sdNVP at birth (infants also received sdNVP and AZT for 7 days), transmission at 4 weeks was $3.1 \%{ }^{31}$ In Kenya, Burkina Faso and South Africa, among breastfed infants whose mothers had CD4 counts 350-500 and received either triple ARVs or AZT from 28 weeks, there were 11 infections among 335 infants at 6 weeks of age $=3.3 \% .{ }^{26}$ In Thailand, among infants who were formula fed and whose mothers had CD4 counts $>200$ and received AZT from 28 weeks and sdNVP at birth (infants also received sdNVP), there were eight infections among 508 infants $=1.6 \%{ }^{38}$

The peripartum transmission probability for Option A should be less than the probability associated with the WHO 2006 dual prophylaxis (4\%), on the grounds that the option would apply only to mothers with CD4>350, the ARV interventions would be started earlier in pregnancy from 14 weeks gestation and the extended postnatal ARV regimen (AZT or NVP for 6 weeks) to infants would provide additional protection. Hence, a peripartum transmission probability of $2 \%$ would be plausible and consistent with published data.

Four randomised studies reported the efficacy of nevirapine given to infants to reduce the risk of HIV transmission while breast feeding. Two of the studies provided only limited data. ${ }^{36}{ }^{40}$ The two other studies reported transmission rates from HIV-exposed, uninfected infants recruited and randomised postdelivery and who received nevirapine for up to 6 months while breast feeding. ${ }^{41}{ }^{42}$ A simple median value from these studies would be inappropriate as both studies, included mothers with CD4 counts 200-350. Considering the population for whom Option A is recommended ( $>350$ cells $/ \mathrm{ml}$ ), an estimate of $0.2 \%$ per month of breast feeding is consistent with available data.

\section{Transmission probabilities in the context of Option B regimen}

As with Option A, no studies report transmission outcomes when all elements of Option B are implemented in the target population. For example, the Kesho Bora study provided the same ARV interventions but started only at 28 weeks and did not include 6 weeks extended postpartum ARVs to the infants. Several studies provide peripartum transmission probabilities associated with triple ARV prophylaxis in women who are not eligible for lifelong ART and are summarised in the online supplementary table $1 .{ }^{26}{ }^{43-46}$ The range of transmission probabilities was $0.9 \%-2.9 \%$. A peripartum transmission probability of $2 \%$ would be consistent with an earlier initiation of ARVs combined with the postpartum infant intervention.

Three randomised trials and two non-randomised, intervention studies provided data on postpartum transmission. ${ }^{26} 41$ 45-47 Population characteristics were significantly different and monthly postnatal transmission probabilities were calculated based on data provided. Additional discussion of the studies, interventions implemented and reported transmission are included in the working paper (online supplementary appendix). The range of estimates of postnatal HIV transmission was $0.063 \%-0.53 \%$ per month of breast feeding. These may be even less if mothers with CD4 $>500$ were included. For mothers with CD4 counts $>350$, an estimate of $0.2 \%$ per month of breast feeding would be consistent with published literature.

\section{Transmission probabilities in the context of lifelong ART}

Two reports from the UK, Ireland and France and three studies from west, south and east Africa provide data that inform this estimate. Studies from Europe indicate peripartum transmission among women on ART to be around $1 \% .{ }^{43} 44$ These mothers generally had caesarean section and no infants were breast fed. ART was sometimes started prior to conception. African studies report a wider range of transmission probabilities $(0.6 \%-3.7 \%){ }^{26} 31245$ When lifelong ART is started in pregnant women with CD4 counts less than 350 cells $/ \mathrm{ml}$ then a peripartum transmission probability of $2 \%$ or less is expected.

Limited data were available on transmission risks when women were on ART prior to conception. In the UK and Ireland, among infants born by caesarean section to HIV-infected mothers on triple ARVs from conception the peripartum transmission probability was $0.1 \%{ }^{44}$ In Botswana and South Africa, programmes reported peripartum transmission probabilities of $0.3 \%$ and $0.7 \%$ among infants who were not breast fed and whose mothers were on lifelong ART prior to conception. ${ }^{48}{ }^{49}$ In two randomised studies conducted in Botswana, Kenya, Burkina Faso and South Africa, among women who started ARV interventions during pregnancy, but where there was good ARV adherence and effective viral suppression, peripartum transmission probabilities were $0.63 \%$ and $1.3 \%{ }^{26}{ }^{25}$

These probabilities are consistent with the strong relationship between low transmission and longer duration of ARV treatment in pregnancy, good adherence to ARVs and effective viral suppression. ${ }^{50}$ In the context of HIV-infected mothers being on lifelong ART initiated prior to conception, peripartum transmission probability is estimated to be $0.5 \%$.

There was considerable variation in the reported estimates of postnatal transmission from African studies in mothers on ART. ${ }^{31} 3245-47$ These ranged from $0 \%$ to $0.42 \%$ per month of breast feeding. Effective viral suppression was associated with low postnatal transmission. Differences in reported transmission probabilities may reflect longer intervals between ART initiation and birth or initiation of breast feeding, and different rates of ARV adherence and associated viral suppression. Although the median value of reported transmission probabilities was $0.16 \%$ per month of breast feeding, it would be inconsistent, on therapeutic and programmatic grounds, for postnatal transmission rates in mothers eligible for, and starting ART during pregnancy, to be lower than transmission rates 
among mothers with CD4 counts greater than 350 cells $/ \mathrm{ml}$ and starting similar ARV interventions that are recommended as part of Option B.

Data from four studies help to inform the postnatal transmission probability among women who are on ART prior to conception. In Botswana, no postnatal transmissions occurred in a randomised trial among breastfeeding HIV-infected mothers who received one of two triple ARV regimens and viral suppression was achieved in more than $94 \%$ mothers. ${ }^{45}$ In Rwanda, there were $0.5 \%$ transmission over 8 months breastfeeding exposure $(=0.063 \%$ per month) among mothers with CD4 $<350$ cells $/ \mathrm{ml}$ who started on lifelong ART (D4T, 3TC and NVP) and among mothers with higher CD4 (>350 cells/ml) who received AZT, 3TC and efavirenz. ${ }^{46}$ In Burkina Faso, Kenya and South Africa, when there was effective viral suppression among mothers who received triple ARVs as prophylaxis, postnatal transmission probability at 6 months was $0.9 \%{ }^{26}$ In Malawi, differences in early transmission probabilities in intervention arms suggest that a small percentage of postnatal transmission could be additionally prevented by earlier ART initiation. $^{41}$

In the context of infants also receiving ARV prophylaxis for the first 6 weeks post partum, the postnatal transmission probability is likely to be similar in women who have been on ART from before conception and in women who start ART during pregnancy. In light of these data, the Working group estimated that in the context of HIV-infected mothers being on lifelong ART initiated prior to conception the postnatal transmission probability would be $0.16 \%$ transmission per month of breast feeding.

\section{DISCUSSION}

The above tables (and online supplementary table 1) summarise the HIV transmission probabilities applied in the AIM module of Spectrum since April 2011 (V.4.3). The source data and process by which the transmission probabilities were estimated, including the range of reported MTCT probabilities by timing of infection, maternal CD4 count and ARV intervention, are described and justified. Providing this information enables better understanding and interpretation of Spectrum modelled transmission rates and the uncertainties around those estimates.

For the first time, Spectrum includes monthly transmission probabilities for the breastfeeding period by ARV intervention and whether women have recently acquired HIV infection. The probabilities are frequently derived from randomised controlled trials and do not necessarily reflect the programmatic challenges of delivering interventions to populations in different geographic or cultural contexts. These factors will be included in modelled estimates as determinants of coverage rather than therapeutic effect of an ARV regimen in individual mothers and infants.

For incident infections occurring when mothers are breast feeding, an overall transmission rate is estimated rather than a monthly transmission probability. The available data tend not to support a relationship between duration of breast feeding and the risk of HIV transmission to the infant among women acquiring HIV during lactation. ${ }^{17}$ The lack of relationship is plausible. It may be that an extraordinarily high maternal viral load during incident infection results in most infections occurring during the first weeks of breastfeeding exposure. This period of high risk may only be present for 1-2 months and then may decline to a low risk level. ${ }^{17}$ It may therefore be inappropriate to apply an average monthly hazard risk over the duration of breast feeding in this case. In the largest of the studies, which also provided the greatest detail on timing of maternal seroconversion and infant infection, about 14\% (95\% CI $10.7 \%$ to $19.0 \%$ ) of breastfeeding infants born to mothers who converted at any time in the postnatal period became infected within 6 months after maternal infection. In the subgroup of mothers who were known to have seroconverted within the preceding 90 days, $24 \%$ (95\% CI $14.15 \%$ to $39.48 \%$ ) of breastfeeding infants became infected within 6 months of maternal infection. ${ }^{17}$

There are several limitations to the estimates of transmission probabilities. Estimating the transmission probabilities when mothers and infants receive PMTCT Option A or B is complicated since no single study implemented all elements of either option. The transmission probabilities for these regimens are therefore extrapolated from findings reported in a number of studies. For interventions such as single dose nevirapine or WHO 2006 dual prophylaxis, transmission probabilities are not disaggregated by maternal CD4 count. It is assumed that these probabilities will only be applied to historical populations where CD4 counts were generally not available, and to regimens that are no longer recommended. A transmission probability is included however for the scenario where PMTCT Option A is provided to mothers who may have a CD4 count between 200 and $350 \mathrm{cells} / \mathrm{ml}$. Even though this is not recommended, it was considered a likely scenario-especially in settings where CD4 counts are not routinely available (in 2010, only $30 \%$ pregnant HIV-infected women were assessed for ART eligibility by CD4 measurement ${ }^{51}$ ) —and therefore reasonable to include. A simple method was used to derive the monthly postnatal transmission probabilities which did not take into account whether the child was infected in the previous month. For very small probabilities this will not have an important effect. However when multiplied over several months the result could be significant. Future versions of Spectrum should allow for more decimal places in the monthly transmission probability.

The transmission probability during breast feeding is assumed to be the same among women who are on lifelong ART prenatally or if started on ART during pregnancy. Compared with women who start ART during pregnancy, initiating HIV-infected women on ART before pregnancy is likely to have a greater impact on peripartum transmission than transmission through breast feeding. The postnatal viral load of HIV-infected lactating mothers is not likely to be significantly different whether ART is started before pregnancy or whether it is started within the first or second trimester. However, we are unaware of evidence to inform this transmission risk and additional data are needed to validate these assumptions.

As much as it is important to apply the most accurate transmission probabilities, the quality of input data from national programmes must be optimised for the model estimate to be valid. In particular, it is essential that good quality data differentiate the number of women receiving ARVs, either as prophylaxis or ART, and on the distribution of CD4 counts with different populations. This is needed especially in settings where Option A is implemented and where the transmission probability will be higher than expected if women with lower CD4 counts are not identified and managed appropriately. Where information on the duration of breast feeding among women in the PMTCT programme is not available, Spectrum applies breastfeeding rates and duration to mothers who are HIV-infected according to patterns reported in national Demographic and Health Surveys. This may not be an appropriate assumption for women in the PMTCT programme as 
these women might preferentially reduce breastfeeding duration. This highlights the need for better data on infant feeding practices and adherence to recommended ARV interventions during this time period as well as during pregnancy. Last, in order to better estimate and interpret modelled outcomes, it is important for empiric transmission rates to be measured through population-based sampling.

The transmission probabilities presented in table 1 and the new infection rates that will be estimated from the models do not reflect all the considerations on which WHO clinical recommendations need to be based. For example, transmission probabilities do not reflect the full risk-benefit opportunities of mothers being able to breast feed their infant compared with using replacement feeds. The value of interventions to reduce HIV transmission can only be judged when understood in the context of overall maternal and child health and survival. For example, the value of an intervention may be greatly increased if it also improves maternal health and survival or reduces the risk of HIV transmission to another adult. The Spectrum model does not include any measure of viral resistance or drug safety and the consequences of these adverse events on population outcomes is not therefore evident. It is quite likely that new data on other benefits (and potential risks) of ARVs will become available in the next few years and will significantly influence PMTCT recommendations. It is less likely, however, that new ARV interventions with greater therapeutic efficacy to decrease HIV transmission will be developed in the near future.

The Global Plan Towards the Elimination of New HIV Infections among Children and Keeping Their Mothers Alive is the most ambitious initiative for improving the health and survival of HIV-infected mothers and their children in the history of the HIV epidemic. ${ }^{6}$ It is therefore essential to be able to track, with confidence, progress towards the targets. Applying rigorously-derived transmission probabilities in models such as Spectrum facilitates interpretation of national and global estimates and trends of the number of children becoming infected with HIV, and will allow better comparisons with programmatic data or estimates from other models.

\section{Key messages}

- Publishing the mother-to-child transmission probabilities used in Spectrum allows other modellers to understand and compare their results with Spectrum results.

- Peripartum HIV transmission probabilities depend on maternal CD4, antiretroviral drug regimen, incident or prevalent maternal HIV infection and range from $37 \%$ to $0.5 \%$.

- Postnatal HIV transmission probabilities in mothers with prevalent infection range from $1.57 \%$ to $0.16 \%$ per month of breast feeding. With incident infection, $28 \%$ transmission is expected irrespective of duration of breast feeding.

Acknowledgements The authors thank Dr Elaine Abrams for her review of an earlier draft of this paper.

Contributors NR and MM reviewed the data, developed the tables and drafted the manuscript. RB, LK, TC and LM reviewed the data and the manuscript.

Disclaimer N Rollins is a staff member of WHO. The authors alone are responsible for the views expressed in this publication and they do not necessarily represent the decisions, policy or views of WHO.

\section{Competing interests None}

Provenance and peer review Commissioned; externally peer reviewed.

\section{REFERENCES}

1. UNAIDS. UNAIDS Report on the global AIDS epidemic 2010. http://www.unaids org/globalreport/Global report.htm (last accessed 21 Feb 2011).

2. Barker PM, Mphatswe W, Rollins N. Antiretroviral drugs in the cupboard are not enough: the impact of health systems' performance on mother-to-child transmission of HIV. J Acquir Immune Defic Syndr 2011;56:e45-8.

3. Stover J, McKinnon R, Winfrey B. Spectrum: a model platform for linking maternal and child survival interventions with AIDS, family planning and demographic projections. Int J Epidemiol 2010;39(Suppl 1):i7-10.

4. Stover J, Johnson P, Hallett T, et al. The Spectrum projection package: improvements in estimating incidence by age and sex, mother-to-child transmission, HIV progression in children and double orphans. Sex Transm Infect 2010;86(Suppl 2):ii16-21.

5. World Health Organization. Guidelines on Antiretroviral drugs for treating pregnant women and preventing HIV infections in infants: Recommendations for a public health approach. 2010.http://www.who.int/hiv/pub/mtct/antiretroviral2010/en/ (last accessed Feb 2011).

6. UNAIDS. Countdown To Zero. Global Plan Towards the Elimination of New HIV Infections among Children by 2015 and Keeping Their Mothers Alive. 2011-2015. http://www unaids org/en/media/unaids/contentassets/documents/unaidspublication/ 2011/20110609_JC2137_Global-Plan-Elimination-HIV-Children_en pdf 2011 (accessed 1 Aug 2012).

7. WHO. Antiretroviral drugs for treating pregnant women and preventing HIV infection in infants in resource-limited settings. Towards Universal Access. Recommendations for a public health approach. http://www.who.int/hiv/pub/guidelines/en/index.html. http://www who int/hiv/pub/guidelines/en/index html 2006

8. Rollins N, Little K, Mzolo S, et al. Surveillance of mother-to-child transmission prevention programmes at immunization clinics: the case for universal screening. AIDS 2007;21:1341-7

9. Birkhead GS, Pulver WP, Warren BL, et al. Acquiring human immunodeficiency virus during pregnancy and mother-to-child transmission in New York: 2002-2006. Obstet Gynecol 2010;115:1247-55.

10. Roongpisuthipong A, Siriwasin W, Simonds RJ, et al. HIV seroconversion during pregnancy and risk for mother-to-infant transmission. J Acquir Immune Defic Syndr 2001;26:348-51.

11. Tovo PA, Palomba E, Gabiano C, et al. Human immunodeficiency virus type 1 (HIV-1) seroconversion during pregnancy does not increase the risk of perinatal transmission. Br J Obstet Gynaecol 1991;98:940-2.

12. Van de Perre $\mathbf{P}$, Simonon A, Msellati $\mathbf{P}$, et al. Postnatal transmission of human immunodeficiency virus type 1 from mother to infant. A prospective cohort study in Kigali, Rwanda. N Engl J Med 1991;325:593-8.

13. Dunn DT, Newell ML, Ades AE, et al. Risk of human immunodeficiency virus type 1 transmission through breastfeeding. Lancet 1992;340:585-8.

14. Palasanthiran P, Ziegler JB, Stewart GJ, et al. Breast-feeding during primary maternal human immunodeficiency virus infection and risk of transmission from mother to infant. J Infect Dis 1993;167:441-4.

15. Ekpini ER, Wiktor SZ, Satten GA, et al. Late postnatal mother-to-child transmission of HIV-1 in Abidjan, Cote d'Ivoire. Lancet 1997;349:1054-9.

16. Liang $\mathbf{K}$, Gui $X$, Zhang $Y Z$, et al. A case series of 104 women infected with HIV-1 via blood transfusion postnatally: high rate of HIV-1 transmission to infants through breast-feeding. J Infect Dis 2009;200:682-6.

17. Humphrey $\mathbf{J H}$, Marinda E, Mutasa K, et al. Mother to child transmission of HIV among Zimbabwean women who seroconverted postnatally: prospective cohort study. BMJ 2010;341:c6580.

18. PETRA Study Team. Efficacy of three short-course regimens of zidovudine and lamivudine in preventing early and late transmission of HIV-1 from mother to child in Tanzania, South Africa, and Uganda (Petra study): a randomised, double-blind, placebo-controlled trial. Lancet 2002;359:1178-86.

19. Connor EM, Sperling RS, Gelber R, et al. Reduction of maternal-infant transmission of human immunodeficiency virus type 1 with zidovudine treatment. Pediatric AIDS Clinical Trials Group Protocol 076 Study Group. N Engl J Med 1994;331:1173-80.

20. Shaffer N, Chuachoowong R, Mock PA, et al. Short-course zidovudine for perinatal HIV-1 transmission in Bangkok, Thailand: a randomised controlled trial. Bangkok Collaborative Perinatal HIV Transmission Study Group. Lancet 1999;353:773-80.

21. Wiktor SZ, Ekpini E, Karon JM, et al. Short-course oral zidovudine for prevention of mother-to-child transmission of HIV-1 in Abidjan, Cote d'Ivoire: a randomised trial. Lancet 1999;353:781-5.

22. Dabis F, Msellati P, Meda N, et al. 6-month efficacy, tolerance, and acceptability of a short regimen of oral zidovudine to reduce vertical transmission of HIV in breastfed children in Cote d'Ivoire and Burkina Faso: a double-blind placebo-controlled multicentre trial. DITRAME Study Group. DIminution de la Transmission Mere-Enfant. Lancet 1999;353:786-92. 
23. Leroy V, Sakarovitch C, Cortina-Borja M, et al. Is there a difference in the efficacy of peripartum antiretroviral regimens in reducing mother-to-child transmission of HIV in Africa? AIDS 2005;19:1865-75.

24. Mayaux MJ, Blanche S, Rouzioux C, et al. Maternal factors associated with perinatal HIV-1 transmission: the French Cohort Study: 7 years of follow-up observation. The French Pediatric HIV Infection Study Group. J Acquir Immune Defic Syndr Hum Retrovirol 1995;8:188-94.

25. Kuhn L, Aldrovandi GM, Sinkala M, et al. Potential impact of new WHO criteria for antiretroviral treatment for prevention of mother-to- child HIV transmission. AIDS 2010;24:1374-7.

26. Kesho Bora Study Group. Triple antiretroviral compared with zidovudine and single-dose nevirapine prophylaxis during pregnancy and breastfeeding for prevention of mother-to-child transmission of HIV-1 (Kesho Bora study): a randomised controlled trial. Lancet Infect Dis 2011:11:171-80.

27. Mofenson L, Taha TE, Li Q, et al. Infant extended antiretroviral (ARV) prophylaxis is effective in preventing postnatal mother-to-child HIV transmission (MTCT) at all maternal CD4 counts. TUPEC053. 5th IAS Conference on HIV Pathogenesis, Treatment and Prevention; 19-22 July 2009, Cape Town, South Africa

28. Coutsoudis A, Dabis F, Fawzi W, et al. Late postnatal transmission of HIV-1 in breast-fed children: an individual patient data meta-analysis. J Infect Dis 2004;189:2154-66.

29. Iliff PJ, Piwoz EG, Tavengwa NV, et al. Early exclusive breastfeeding reduces the risk of postnatal HIV-1 transmission and increases HIV-free survival. AIDS 2005;19:699-708.

30. Coovadia HM, Rollins NC, Bland RM, et al. Mother-to-child transmission of HIV-1 infection during exclusive breastfeeding in the first 6 months of life: an intervention cohort study. Lancet 2007;369:1107-16.

31. Tonwe-Gold B, Ekouevi DK, Viho I, et al. Antiretroviral treatment and prevention of peripartum and postnatal HIV transmission in West Africa: evaluation of a two-tiered approach. PLoS Med 2007;4:e257.

32. Kesho Bora Study Group. Eighteen-month follow-up of HIV-1-infected mothers and their children enrolled in the Kesho Bora study observational cohorts. J Acquir Immune Defic Syndr 2010;54:533-41.

33. Guay LA, Musoke P, Fleming T, et al. Intrapartum and neonatal single-dose nevirapine compared with zidovudine for prevention of mother-to-child transmission of HIV-1 in Kampala, Uganda: HIVNET 012 randomised trial. Lancet 1999:354:795-802

34. Jackson JB, Musoke P, Fleming T, et al. Intrapartum and neonatal single-dose nevirapine compared with zidovudine for prevention of mother-to-child transmission of HIV-1 in Kampala, Uganda: 18-month follow-up of the HIVNET 012 randomised trial. Lancet 2003;362:859-68

35. Moodley D, Moodley J, Coovadia $\mathrm{H}$, et al. A multicenter randomized controlled tria of nevirapine versus a combination of zidovudine and lamivudine to reduce intrapartum and early postpartum mother-to-child transmission of human immunodeficiency virus type 1. J Infect Dis 2003;187:725-35.

36. SWEN Study TeamBedri A, Gudetta B, Sehak A, et al. Extended-dose nevirapine to 6 weeks of age for infants to prevent HIV transmission via breastfeeding in
Ethiopia, India, and Uganda: an analysis of three randomised controlled trials. Lancet 2008;372:300-13

37. Dabis F, Bequet L, Ekouevi DK, et al. Field efficacy of zidovudine, lamivudine and single-dose nevirapine to prevent peripartum HIV transmission. AIDS 2005; 19:309-18.

38. Lallemant $\mathbf{M}$, Jourdain $\mathrm{G}$, Le Coeur $\mathbf{S}$, et al. Single-dose perinatal nevirapine plus standard zidovudine to prevent mother-to-child transmission of HIV-1 in Thailand N Engl J Med 2004;351:217-28.

39. Shapiro RL, Thior I, Gilbert PB, et al. Maternal single-dose nevirapine versus placebo as part of an antiretroviral strategy to prevent mother-to-child HIV transmission in Botswana. AIDS 2006;20:1281-8.

40. Kumwenda NI, Hoover DR, Mofenson LM, et al. Extended antiretroviral prophylaxis to reduce breast-milk HIV-1 transmission. N Engl J Med 2008;359:119-29.

41. Chasela CS, Hudgens MG, Jamieson DJ, et al. Maternal or infant antiretroviral drugs to reduce HIV-1 transmission. N Engl J Med 2010;362:2271-81.

42. Coovadia HM, Brown ER, Fowler MG, et al. Efficacy and safety of an extended nevirapine regimen in infant children of breastfeeding mothers with HIV-1 infection for prevention of postnatal HIV-1 transmission (HPTN 046): a randomised, double-blind, placebo-controlled trial. Lancet 2012;379:221-8.

43. Tubiana R, Le CJ, Rouzioux C, et al. Factors associated with mother-to-child transmission of HIV-1 despite a maternal viral load \&lt; 500 copies/ml at delivery: a case-control study nested in the French perinatal cohort (EPF-ANRS C01). Clin Infect Dis 2010;50:585-96.

44. Townsend CL, Cortina-Borja M, Peckham CS, et al. Low rates of mother-to-child transmission of HIV following effective pregnancy interventions in the United Kingdom and Ireland, 2000-2006. AIDS 2008;22:973-81.

45. Shapiro RL, Hughes MD, Ogwu A, et al. Antiretroviral regimens in pregnancy and breast-feeding in Botswana. N Engl J Med 2010;362:2282-94.

46. Peltier CA, Ndayisaba GF, Lepage P, et al. Breastfeeding with maternal antiretroviral therapy or formula feeding to prevent HIV postnatal mother-to-child transmission in Rwanda. AIDS 2009:23:2415-23.

47. Thomas TK, Masaba R, Borkowf CB, et al. Triple-antiretroviral prophylaxis to prevent mother-to-child HIV transmission through breastfeeding - the Kisumu Breastfeeding Study, Kenya: a clinical trial. PLoS Med 2011;8:e1001015.

48. Hoffman RM, Black V, Technau $\mathrm{K}$, et al. Effects of highly active antiretroviral therapy duration and regimen on risk for mother-to-child transmission of HIV in Johannesburg, South Africa. J Acquir Immune Defic Syndr 2010;54:35-41.

49. Botswana Ministry of Health. Report from the national program for Early Infant Diagnosis of HIV. Gaborone, Botswana: Ministry of Health, Department of HIV/AIDS Prevention and Care, 2011.

50. Sturt AS, Dokubo EK, Sint TT. Antiretroviral therapy (ART) for treating HIV infection in ART-eligible pregnant women. Cochrane Database Syst Rev 2010;(3):CD008440.

51. World Health Organization, UNICEF, and UNAIDS. Progress report 2011: global HIV/AIDS response. Epidemic update and health sector progress towards universal access, 2011 